\title{
GEOMETRICALLY NONLINEAR VIBRATIONS OF THIN VISCO-ELASTIC PERIODIC PLATES ON A FOUNDATION WITH DAMPING: NON-ASYMPTOTIC MODELLING
}

\section{JAROSEAW JĘDRYSIAK}

Lodz University of Technology, Department of Structural Mechanics, Eódź, Poland

e-mail: jarek@p.lodz.pl

\begin{abstract}
The objects under consideration are thin visco-elastic periodic plates with moderately large deflections. Geometrically nonlinear vibrations of these plates are investigated. In order to take into account the effect of microstructure size on behaviour of these plates a non-asymptotic modelling method is proposed. Using this method, called the tolerance modelling, model equations with constant coefficients involving terms dependent on the microstructure size can be derived. In this paper, only theoretical considerations of the problem of nonlinear vibrations of thin visco-elastic periodic plates resting on a foundation with damping are presented.
\end{abstract}

Keywords: thin visco-elastic periodic plates, nonlinear vibrations, effect of microstructure size, analytical tolerance modelling

\section{Introduction}

In this paper, thin visco-elastic plates with a periodic structure in planes parallel to the plate midplane, interacting with a periodically heterogeneous foundation are considered. These plates consist of many identical small elements, called periodicity cells (they are distinguished by dotted lines in Fig. 1). Plates of this kind can have deflections of the order of their thickness. Dynamic problems of these plates are described by nonlinear partial differential equations with coefficients being highly oscillating, periodic and non-continuous functions of $x_{1}, x_{2}$. Hence, these equations are not a good tool to analyse various special problems of the plates under consideration. In order to obtain governing equations with constant coefficients, various simplified approaches are proposed, which introduce effective plate properties. Amongst them, it is necessary to mention those based on the asymptotic homogenization, see Kohn and Vogelius (1984). Unfortunately, the governing equations of these models usually neglect the effect of the microstructure size on the plate behaviour.

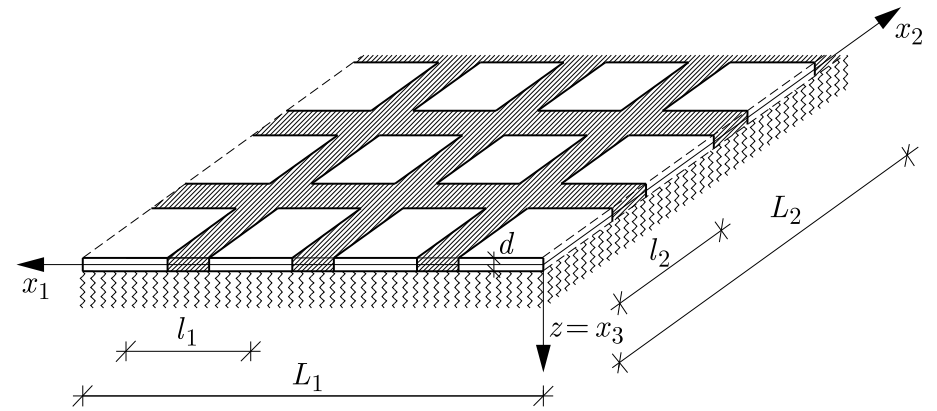

Fig. 1. A fragment of a thin periodic plate on a foundation under consideration 
Other various methods are also applied to describe different mechanical problems of periodic structures and composites. Multiscale models were applied to describe composite materials reinforced by micro-particles by Lurie et al. (2005); the two-scale asymptotic homogenization method was used to analyse honeycomb sandwich composite shells by Saha et al. (2007); a relationship between the 3D and the homogenised Euler-Bernoulli beam limit was shown by Dallot et al. (2009), where the homogenisation procedure was also justified using the asymptotic expansion method. Results suggesting the relevance of the proposed algorithm towards the efficient multiscale modelling of periodic materials such as woven composites were obtained by De Carvalho et al. (2011); a two-dimensional analytical solution of a multilayered rectangular plate with a small periodic structure along one in-plane direction was obtained by He et al. (2013), where the two-scale asymptotic expansion method was employed to develop a homogenized model of each layer in the plate and then the state-space approach was used. Heterogeneous plates were investigated by Schmitz and Horst (2014) using a finite element unit-cell method. The two-dimensional stationary temperature distribution in a periodically stratified composite layer was analysed by Matysiak and Perkowski (2014) within the framework of the homogenized model with microlocal parameters. An asymptotic dispersive method for description of the problem of shear-wave propagation in a laminated composite was proposed by Brito-Santana et al. (2015).

Mechanical problems of thin plates under moderately large deflections are described by the known geometrically nonlinear equations presented by e.g. Timoshenko and Woinowsky-Krieger (1959) and Woźniak (2001). Equations of von Kármán-type plates were derived from equations of the three-dimensional nonlinear continuum mechanics by Meenen and Altenbach (2001). Bending problems of such plates can be analysed using various methods, e.g. proposed by Levy (1942) or Timoshenko and Woinowsky-Krieger (1959). However, other new or modified methods are also presented in a lot of papers. Some of them are mentioned below. An asymptotic approach for thin rectangular plates with variable thickness clamped on all edges was used by Huang (2004). Theoretical, numerical and experimental analysis of the stability and ultimate load of multi-cell thin-walled columns of rectangular cross-sections was shown by Królak et al. (2009). Teter (2011) analysed the dynamic critical load for buckling of columns, but global and local buckling of sandwich beams and plates was examined by Jasion et al. (2012). The nonlinear bending behaviour of moderately thick functionally graded plates on a two-parameter elastic foundation was studied by Golmakani and Alamatian (2013), where the dynamic relaxation method and the finite difference discretization technique were used to solve equations based on the first order shear deformation theory and von Kármán theory.

Problems of nonlinear vibrations and/or visco-elastic damping of composite structures such as beams, plates and shells, are considered by many researchers, applying various methods. The influence of damping and/or stiffness on vibrations of nonlinear periodic plates was shown by Reinhall and Miles (1989). Large amplitude flexural vibration characteristics of composite plates using von Kármán's assumptions and Galerkin's method were obtained by Singha et al. (2009). Geometrically nonlinear vibrations of free-edge circular plates with geometric imperfections described by von Kármán equations with using an expansion onto the eigenmode basis of the perfect plate to discretise the equations of motion were analysed by Camier et al. (2009). An approximate frequency equation of clamped visco-elastic rectangular plates with thickness variations was derived by using the Rayleigh-Ritz technique by Gupta et al. (2009). MagnuckaBlandzi (2010) carried out a certain nonlinear analysis of dynamic stability of a circular plate. Some oscillations of visco-elastic Timoshenko beams were investigated by Manevich and Kołakowski (2011). The variational method was used in nonlinear free vibration and post-buckling analysis of functionally graded beams resting on a nonlinear elastic foundation by Fallah and Aghdam (2011). Damping and forced vibrations of three-layered laminated composite beams described in the framework of the higher-order zig-zag theories were investigated by Youzera et al. (2012). Nonlinear free vibrations of orthotropic shells with variable thickness were analysed 
by Awrejcewicz et al. (2013). Lei et al. (2013) used a transfer function method to obtain a closed-form and uniform solution for damped visco-elastic vibrations of Euler-Bernoulli beams. A linearized updated mode method was applied to solve nonlinear equations of geometrically nonlinear free vibrations of laminated composite rectangular plates with curvilinear fibers by Houmat (2013). Natural frequencies of free vibrations for functionally graded annular plates resting on a Winkler's foundation were predicted using the differential quadrature method and the Chebyshev collocation technique by Yajuvindra and Lal (2013). Yaghoobi and Torabi (2013) presented large amplitude vibrations of functionally graded beams on a nonlinear elastic foundation. Nonlinear bending vibrations of sandwich plates with a visco-elastic core were investigated by Mahmoudkhani et al. (2014), where the 5th-order method of multiple scales was applied to solve the equations of motion.

Usually, those proposed modelling approaches for microstructured media lead to governing equations neglecting the effect of the microstructure size which can play a crucial role in dynamical problems of such media, e.g. for periodic plates under consideration, see Jędrysiak (2003, 2009). In order to take into account this effect, some special methods are adopted sometimes to analyse particular problems. For example, Zhou et al. (2014) investigated the problem of free flexural vibration of periodic stiffened thin plates using Bloch's theorem and the center finite difference method.

However, in order to obtain equations of the model, which describe the aforementioned effect, new non-asymptotic models of thin periodic plates based on the nonlinear theory were proposed by Domagalski and Jędrysiak $(2012,2015)$. These models are called the tolerance models and are obtained in the framework of a certain general modelling approach called the tolerance averaging technique, see Woźniak et al. $(2008,2010)$. The derived equations, in contrary to the exact ones, have constant coefficients. Some of them explicitly depend on the characteristic size of the periodicity cell.

The tolerance method is general and is useful to model various problems described by differential equations with highly oscillating non-continuous functional coefficients. It can be applied in analysis of various thermo-mechanical problems of microheterogeneous solids and structures. Some applications of this method for different periodic structures were presented in a series of papers, e.g. for dynamics of plane periodic structures by Wierzbicki and Woźniak (2000); for dynamics with near-boundary phenomena in stratified layers by Wierzbicki et al. (2001); for vibrations of periodic wavy-type plates by Michalak (2001); for thin plates reinforced by a system of periodic stiffeners by Nagórko and Woźniak (2002); for stability problems of periodic thin plates by Jędrysiak (2000); for stability analysis of periodic shells by Tomczyk (2007); for vibrations of periodic plates by Jędrysiak (2003, 2009); for dynamics problems of medium thickness plates on a periodic foundation by Jędrysiak and Paś (2014); for vibrations of thin functionally graded plates with plate thickness small comparing to the microstructure size by Kaźmierczak and Jędrysiak (2011) and for stability of such plates by Jędrysiak and Michalak (2011); for vibrations of thin functionally graded plates with the microstructure size of an order of the plate thickness by Jędrysiak (2013); for stability of thin functionally graded annular plates on an elastic heterogeneous subsoil by Perliński et al. (2014). Moreover, the tolerance method is also used to analyse damped vibrations of periodic plate strips by Marczak and Jędrysiak (2014) and nonlinear vibrations of periodic beams resting on a visco-elastic foundation by Domagalski and Jędrysiak (2014). An extended list of papers can be found in the books by Woźniak et al. $(2008,2010)$.

The main aim of this theoretical contribution is to formulate and discuss the nonlinear tolerance and asymptotic models of dynamic problems for thin visco-elastic periodic plates with moderately large deflections resting on a foundation with damping, on various levels of accuracy. These new tolerance models are an extension and generalization of the tolerance models 
presented and applied by Domagalski and Jędrysiak (2012, 2014, 2015), Marczak and Jędrysiak (2014).

\section{Fundamental equations}

Let us denote by $0 x_{1} x_{2} x_{3}$ the orthogonal Cartesian co-ordinate system in the physical space and by $t$ the time co-ordinate. Let the subscripts $\alpha, \beta, \ldots(i, j, \ldots)$ run over 1,2 (over $1,2,3$ ) and the indices $A, B, \ldots(a, b, \ldots)$ run over $1, \ldots, N(1, \ldots, n)$. The summation convention holds for all aforementioned indices. Denote also $\mathbf{x} \equiv\left(x_{1}, x_{2}\right)$ and $z \equiv x_{3}$. Let us assume that the undeformed plate occupies the region $\Omega \equiv\{(\mathbf{x}, z):-d(\mathbf{x}) / 2<z<d(\mathbf{x}) / 2, \mathbf{x} \in \Pi\}$, where $\Pi$ is the midplane with length dimensions $L_{1}, L_{2}$ along the $x_{1^{-}}$and $x_{2}$-axis, respectively, and $d(\mathbf{x})$ is plate thickness.

It is assumed that plates under consideration have a periodic structure along the $x_{1}$ - and $x_{2}$-axis directions with periods $l_{1}, l_{2}$, respectively, in planes parallel to the plate midplane. The periodicity basic cell on $0 x_{1} x_{2}$ plane is denoted by $\Delta \equiv\left[-l_{1} / 2, l_{1} / 2\right] \times\left[-l_{2} / 2, l_{2} / 2\right]$. It is assumed that the cell size is described by a parameter $l \equiv\left[\left(l_{1}\right)^{2}+\left(l_{2}\right)^{2}\right] 1 / 2$, satisfying the condition $\max (d) \ll l \ll \min \left(L_{1}, L_{2}\right)$. Thus, $l$ will be called the microstructure parameter. Let us denote partial derivatives with respect to a space co-ordinate by $(\cdot)_{, \alpha} \equiv \partial / \partial x_{\alpha}$.

Moreover, thickness $d(\mathbf{x})$ can be a periodic function in $\mathbf{x}$, elastic moduli $a_{i j k l}=a_{i j k l}(\mathbf{x}, z)$ and mass density $\rho=\rho(\mathbf{x}, z)$ can be also periodic functions in $\mathbf{x}$. In general, these plate properties are not assumed to be even functions in $z$. Let $a_{\alpha \beta \gamma \delta}, a_{\alpha} \beta 33, a_{3333}$ be non-zero components of the elastic moduli tensor. Denote $c_{\alpha \beta \gamma \delta} \equiv a_{\alpha \beta \gamma \delta}-a_{\alpha \beta 33} a_{\gamma \delta 33}\left(a_{3333}\right)^{-1}$. Proper visco-elastic moduli are denoted by $\widetilde{c}_{\alpha \beta \gamma \delta}$.

It is also assumed that the periodic plates interact with a periodic visco-elastic foundation which rests on a rigid undeformable base, see Vlasov and Leontiev (1960). A fragment of such a plate is presented in Fig. 1. The heterogeneous foundation is assumed to be periodic in planes parallel to the plate midplane, i.e. along the $x_{1}$ - and $x_{2}$-axis directions with periods $l_{1}$ and $l_{2}$, respectively; however, it has constant properties along the $z$-axis direction. Hence, the foundation properties, i.e. mass density per unit area $\widehat{\mu}=\widehat{\mu}(\mathbf{x})$, Winkler's coefficient $k=k(\mathbf{x})$ and the damping parameter $c=c(\mathbf{x})$ can be periodic functions in $\mathbf{x}=\left(x_{1}, x_{2}\right)$. These foundation parameters can be defined following the book by Vlasov and Leontiev (1960). It is also assumed that the plate cannot be torn off from the foundation.

Denote displacements, strains and stresses by $u_{i}, e_{i j}$ and $s_{i j}$, respectively; virtual displacements and virtual strains by $\bar{u}_{i}$ and $\bar{e}_{i j}$; loadings (along the $z$-axis) by $p$.

Now, the fundamental relations of the nonlinear thin plates theory, see Levy (1942) and Woźniak et al. (2001), are reminded.

- Kinematic assumptions of thin plates

$$
u_{\alpha}(\mathbf{x}, z, t)=u_{\alpha}^{0}(\mathbf{x}, t)-z \partial_{\alpha} w(\mathbf{x}, t) \quad u_{3}(\mathbf{x}, z, t)=w(\mathbf{x}, t)
$$

with $w(\mathbf{x}, t)$ as the deflection of the midplane, $u_{\alpha}^{0}(\mathbf{x}, t)$ as the in-plane displacement. Similarly, these are for virtual displacements

$$
\bar{u}_{\alpha}(\mathbf{x}, z)=\bar{u}_{\alpha}^{0}(\mathbf{x})-z \partial_{\alpha} \bar{w}(\mathbf{x}) \quad \bar{u}_{3}(\mathbf{x}, z)=\bar{w}(\mathbf{x})
$$

- Strain-displacement relations

$$
e_{\alpha \beta}=u_{(\alpha, \beta)}+\frac{1}{2} u_{3, \alpha} u_{3, \beta}
$$


- Stress-strain relations (it is assumed that the plane of elastic symmetry is parallel to the plane $z=0$ )

$$
s_{\alpha \beta}=c_{\alpha \beta \gamma \delta} e_{\gamma \delta}+\widetilde{c}_{\alpha \beta \gamma \delta} \dot{e}_{\gamma \delta}
$$

with

$$
\begin{array}{ll}
c_{\alpha \beta \gamma \delta}=a_{\alpha \beta \gamma \delta}-a_{\alpha \beta 33} a_{33 \gamma \delta} / a_{3333} & c_{\alpha 3 \gamma 3}=a_{\alpha 3 \gamma 3}-a_{\alpha 333} a_{33 \gamma 3} / a_{3333} \\
\widetilde{c}_{\alpha \beta \gamma \delta}=\widetilde{a}_{\alpha \beta \gamma \delta}-\widetilde{a}_{\alpha \beta 33} \widetilde{a}_{33 \gamma \delta} / \widetilde{a}_{3333} & \widetilde{c}_{\alpha 3 \gamma 3}=\widetilde{a}_{\alpha 3 \gamma 3}-\widetilde{a}_{\alpha 333} \widetilde{a}_{33 \gamma 3} / \widetilde{a}_{3333}
\end{array}
$$

- The virtual work equation

$$
\begin{aligned}
& \int_{\Pi} \int_{-d / 2}^{d / 2} \rho \ddot{u}_{i} \bar{u}_{i} d z d a+\int_{\Pi} \int_{-d / 2}^{d / 2} s_{\alpha \beta} \bar{e}_{\alpha \beta} d z d a=\int_{\Pi} p \bar{u}_{3}\left(\mathbf{x}, \frac{d}{2}\right) d a \\
& \quad-\int_{\Pi}\left(k u_{3}+\widehat{\mu} \ddot{u}_{3}+c \dot{u}_{3}\right) \bar{u}_{3}\left(\mathbf{x},-\frac{d}{2}\right) d a
\end{aligned}
$$

is satisfied for arbitrary virtual displacements (2.2), assuming these displacements neglect the plate boundary; moreover: $d a=d x_{1} d x_{2}$; the virtual displacements are sufficiently regular, independent functions.

The plate properties are periodic functions in $\mathbf{x}$, i.e. stiffness tensors: $b_{\alpha \beta \gamma \delta}, d_{\alpha \beta \gamma \delta}, h_{\alpha \beta \gamma \delta}$, visco-elastic tensors: $\widetilde{b}_{\alpha \beta \gamma \delta}, \widetilde{d}_{\alpha \beta \gamma \delta}, \widetilde{h}_{\alpha \beta \gamma \delta}$, and inertia properties: $\mu, j, i$ are defined as

$$
\begin{array}{lc}
b_{\alpha \beta \gamma \delta}(\mathbf{x})=\int_{-d / 2}^{d / 2} c_{\alpha \beta \gamma \delta}(\mathbf{x}, z) d z & d_{\alpha \beta \gamma \delta}(\mathbf{x})=\int_{-d / 2}^{d / 2} c_{\alpha \beta \gamma \delta}(\mathbf{x}, z) z^{2} d z \\
h_{\alpha \beta \gamma \delta}(\mathbf{x})=\int_{-d / 2}^{d / 2} c_{\alpha \beta \gamma \delta}(\mathbf{x}, z) z d z & \widetilde{b}_{\alpha \beta \gamma \delta}(\mathbf{x})=\int_{-d / 2}^{d / 2} \widetilde{c}_{\alpha \beta \gamma \delta}(\mathbf{x}, z) d z \\
\widetilde{d}_{\alpha \beta \gamma \delta}(\mathbf{x})=\int_{-d / 2}^{d / 2} \widetilde{c}_{\alpha \beta \gamma \delta}(\mathbf{x}, z) z^{2} d z & \widetilde{h}_{\alpha \beta \gamma \delta}(\mathbf{x})=\int_{-d / 2}^{d / 2} \widetilde{c}_{\alpha \beta \gamma \delta}(\mathbf{x}, z) z d z \\
\mu(\mathbf{x})=\int_{-d / 2}^{d / 2} \rho(\mathbf{x}, z) d z & j(\mathbf{x})=\int_{-d / 2}^{d / 2} \rho(\mathbf{x}, z) z^{2} d z \quad i(\mathbf{x})=\int_{-d / 2}^{d / 2} \rho(\mathbf{x}, z) z d z
\end{array}
$$

Using assumptions (2.1)-(2.4) of the nonlinear two-dimensional thin plate theory, applying the divergence theorem and the du Bois-Reymond lemma to equation (2.6), after some manipulations the governing equations of thin visco-elastic plates resting on foundations with damping can be written in the form:

- constitutive equations

$$
\begin{aligned}
m_{\alpha \beta} & =-h_{\alpha \beta \gamma \delta} u_{\gamma, \delta}+d_{\alpha \beta \gamma \delta} w_{, \gamma \delta}-\frac{1}{2} h_{\alpha \beta \gamma \delta} w_{, \gamma} w_{, \delta}-\widetilde{h}_{\alpha \beta \gamma \delta} \dot{u}_{\gamma, \delta}+\widetilde{d}_{\alpha \beta \gamma \delta} \dot{w}_{, \gamma \delta} \\
+ & \frac{1}{2} \widetilde{h}_{\alpha \beta \gamma \delta}\left(\dot{w}_{, \gamma} w_{, \delta}+w_{, \gamma} \dot{w}_{, \delta}\right) \\
n_{\alpha \beta} & =b_{\alpha \beta \gamma \delta} u_{\gamma, \delta}-h_{\alpha \beta \gamma \delta} w_{, \gamma \delta}+\frac{1}{2} b_{\alpha \beta \gamma \delta} w_{, \gamma} w_{, \delta}+\widetilde{b}_{\alpha \beta \gamma \delta} \dot{u}_{\gamma, \delta}-\widetilde{h}_{\alpha \beta \gamma \delta} \dot{w}_{, \gamma \delta} \\
& +\frac{1}{2} \widetilde{b}_{\alpha \beta \gamma \delta}\left(\dot{w}_{, \gamma} w_{, \delta}+w_{, \gamma} \dot{w}_{, \delta}\right)
\end{aligned}
$$


- equilibrium equations

$$
\begin{aligned}
& m_{\alpha \beta, \alpha \beta}-\left(n_{\alpha \beta} w_{, \alpha}\right)_{, \beta}+\mu \ddot{w}-j \ddot{w}_{, \alpha \alpha}+k w+\widehat{\mu} \ddot{w}+c \dot{w}+i \ddot{u}_{\alpha, \alpha}=p \\
& -n_{\alpha \beta, \beta}+\mu \ddot{u}_{\alpha}-i \ddot{w}_{, \alpha}=0
\end{aligned}
$$

or after substituting equations (2.8) into (2.9) as

$$
\begin{aligned}
& \left\{-h_{\alpha \beta \gamma \delta}\left(u_{\gamma, \delta}^{0}+\frac{1}{2} w_{, \gamma} w_{, \delta}\right)+d_{\alpha \beta \gamma \delta} w_{, \gamma \delta}-\widetilde{h}_{\alpha \beta \gamma \delta}\left[\dot{u}_{\gamma, \delta}^{0}-\frac{1}{2}\left(\dot{w}_{, \gamma} w_{, \delta}+w_{, \gamma} \dot{w}_{, \delta}\right)\right]\right. \\
& \left.\quad+\tilde{d}_{\alpha \beta \gamma \delta} \dot{w}_{, \gamma \delta}\right\}_{, \alpha \beta}-\left\{\left[b_{\alpha \beta \gamma \delta}\left(u_{\gamma, \delta}^{0}+\frac{1}{2} w_{, \gamma} w_{, \delta}\right)-h_{\alpha \beta \gamma \delta} w_{, \gamma \delta}\right.\right. \\
& \left.\left.\quad+\widetilde{b}_{\alpha \beta \gamma \delta}\left[\dot{u}_{\gamma, \delta}^{0}+\frac{1}{2}\left(\dot{w}_{, \gamma} w_{, \delta}+w_{, \gamma} \dot{w}_{, \delta}\right)\right]-\widetilde{h}_{\alpha \beta \gamma \delta} \dot{w}_{, \gamma \delta}\right] w_{, \alpha}\right\}_{, \beta} \\
& \quad+\mu \ddot{w}-j \ddot{w}_{, \alpha \alpha}+k w+\widehat{\mu} \ddot{w}+c \dot{w}+i \ddot{u}_{\alpha, \alpha}^{0}=p \\
& -\left\{b_{\alpha \beta \gamma \delta}\left(u_{\gamma, \delta}^{0}+\frac{1}{2} w_{, \gamma} w_{, \delta}\right)-h_{\alpha \beta \gamma \delta} w_{, \gamma \delta}+\widetilde{b}_{\alpha \beta \gamma \delta}\left[\dot{u}_{\gamma, \delta}^{0}+\frac{1}{2}\left(\dot{w}_{, \gamma} w_{, \delta}+w_{, \gamma} \dot{w}_{, \delta}\right)\right]\right. \\
& \left.\quad-\widetilde{h}_{\alpha \beta \gamma \delta} \dot{w}_{, \gamma \delta}\right\}_{, \beta}+\mu \ddot{u}_{\alpha}^{0}-i \ddot{w}_{, \alpha}=0
\end{aligned}
$$

It can be observed that coefficients of equations (2.8) and (2.9) (or (2.10)) can be discontinuous and highly oscillating, periodic functions in $\mathbf{x}$, cf. (2.7). Hence, solutions to these equations are very difficult to obtain.

The main aim of this paper is to propose a replacement of original equations with approximate models, which describe (or not) the information about the microstructure of considered plates by using systems of equations with constant coefficients.

\section{Outline of the tolerance modelling}

\subsection{Introductory concepts}

In the tolerance modelling, certain introductory concepts are used. Following Woźniak et al. (2008, 2010) some of them are reminded below.

A cell at $\mathbf{x} \in \Pi_{\Delta}$ is denoted by $\Delta(\mathbf{x})=\mathbf{x}+\Delta, \Pi_{\Delta}=\{\mathbf{x} \in \Pi: \Delta(\mathbf{x}) \subset \Pi\}$. The fundamental concept of the modelling technique is the averaging operator, defined by

$$
\langle\phi\rangle(\mathbf{x})=\frac{1}{l_{1} l_{2}} \int_{\Delta(\mathbf{x})} f\left(y_{1}, y_{2}\right) d y_{1} d y_{2} \quad \mathbf{x} \in \Pi_{\Delta} \quad \mathbf{y} \in \Delta(\mathbf{x})
$$

for an integrable function $\varphi$. If the function $\varphi$ is periodic in $\mathbf{x}$, its averaged value calculated from (3.1) is constant.

Let $\delta$ be an arbitrary positive number and $X$ be a linear normed space. The tolerance relation $\approx$ for a certain positive constant $\delta$, called the tolerance parameter, is defined by

$$
\left(\forall\left(\mathbf{x}_{1}, \mathbf{x}_{2}\right) \in X^{2}\right) \quad\left[\mathbf{x}_{1} \approx \mathbf{x}_{2} \Leftrightarrow\left\|\mathbf{x}_{1}-\mathbf{x}_{2}\right\|_{X} \leqslant \delta\right]
$$

Let $\partial^{k} \varphi$ denote the $k$-th gradient of the function $\varphi=\varphi(\mathbf{x}), \mathbf{x} \in \Pi, k=0,1, \ldots, \alpha, \alpha \geqslant 0$, and $\partial^{0} \varphi \equiv \varphi$. Let $\widetilde{\phi}^{(k)}(\mathbf{x}, \cdot)$ be a function defined in $\bar{\Pi} \times R^{m}$, and $\delta$ be the tolerance parameter. Introduce also $\Pi_{\mathbf{x}} \equiv \Pi \cap \bigcup_{\mathbf{z} \in \Delta(\mathbf{x})} \Delta(\mathbf{z}), \mathbf{x} \in \bar{\Pi}$. 
The function $\varphi \in H^{\alpha}(\Pi)$ is called the tolerance-periodic function (with respect to cell $\Delta$ and tolerance parameter $\delta), \varphi \in T P_{\delta}^{\alpha}(\Pi, \Delta)$, if for $k=0,1, \ldots, \alpha$, the following conditions are satisfied

(i) $\quad(\forall \mathbf{x} \in \Pi)\left(\exists \widetilde{\phi}^{(k)}(\mathbf{x}, \cdot) \in H^{0}(\Delta)\right)\left[\left\|\partial^{k} \phi\right\|_{\Pi_{\mathbf{x}}}(\cdot)-\widetilde{\phi}^{(k)}(\mathbf{x}, \cdot) \|_{H^{0}\left(\Pi_{\mathbf{x}}\right)} \leqslant \delta\right]$

(ii) $\int_{\Delta(\cdot)} \widetilde{\phi}^{(k)}(\cdot, \mathbf{z}) d \mathbf{z} \in C^{0}(\bar{\Pi})$

The function $\widetilde{\varphi}^{(k)}(\mathbf{x}, \cdot)$ is a periodic approximation of $\partial^{k} \varphi$ in $\Delta(\mathbf{x}), \mathbf{x} \in \Pi, k=0,1, \ldots, \alpha$.

The function $F \in H^{\alpha}(\Pi)$ is a slowly-varying function, $F \in S V_{\delta}^{\alpha}(\Pi, \Delta)$, if

(i) $\quad F \in T P_{\delta}^{\alpha}(\Pi, \Delta)$

(ii) $\quad(\forall \mathbf{x} \in \Pi)\left[\left.\widetilde{F}^{(k)}(\mathbf{x}, \cdot)\right|_{\Delta(\mathbf{x})}=\partial^{k} F(\mathbf{x}), k=0, \ldots, \alpha\right]$

The function $\phi \in H^{\alpha}(\Pi)$ is a highly oscillating function, $\phi \in H O_{\delta}^{\alpha}(\Pi, \Delta)$, if

(i) $\phi \in T P_{\delta}^{\alpha}(\Pi, \Delta)$

(ii) $\quad(\forall \mathbf{x} \in \Pi)\left[\left.\widetilde{\phi}^{(k)}(\mathbf{x}, \cdot)\right|_{\Delta(\mathbf{x})}=\partial^{k} \widetilde{\phi}(\mathbf{x}), k=0, \ldots, \alpha\right]$

(iii) $\quad \forall F \in S V_{\delta}^{\alpha}(\Pi, \Delta) \quad \exists \varphi=\phi F \in T P_{\delta}^{\alpha}(\Pi, \Delta)$

$$
\left.\widetilde{\varphi}^{(k)}(\mathbf{x}, \cdot)\right|_{\Delta(\mathbf{x})}=\left.F(\mathbf{x}) \partial^{k} \widetilde{\phi}(\mathbf{x})\right|_{\Delta(\mathbf{x})}, k=1, \ldots, \alpha
$$

For $\alpha=0$, let us denote $\widetilde{\varphi} \equiv \widetilde{\varphi}^{(0)}$.

Let us introduce two highly oscillating functions defined on $\bar{\Pi}, f(\cdot), g(\cdot), f \in H O_{\delta}^{1}(\Pi, \Delta)$, $g \in H O_{\delta}^{2}(\Pi, \Delta)$.

Let the function $f(\cdot)$ be continuous and have a piecewise continuous and bounded gradient $\partial^{1} f$. The function $f(\cdot)$ is a fluctuation shape function of the 1 st kind, $F S_{\delta}^{1}(\Pi, \Delta)$, if it depends on $l$ as a parameter and the conditions hold

(i) $\quad \partial^{k} f \in O\left(l^{\alpha-k}\right)$ for $k=0, \alpha, \alpha=1, \partial^{0} f \equiv f$

(ii) $\langle f\rangle(\mathbf{x}) \approx 0 \forall \mathbf{x} \in \Pi_{\Delta}$

where $l$ is the microstructure parameter. Condition $(3.6)_{(i i)}$ can be replaced by $\langle\mu f\rangle(\mathbf{x}) \approx 0$ for every $\mathbf{x} \in \Pi_{\Delta}$, where $\mu>0$ is a certain tolerance-periodic function.

However, let $g(\cdot)$ be a continuous function together with the gradient $\partial^{1} g$ and with the piecewise continuous and bounded gradient $\partial^{2} g$. The function $g(\cdot)$ is a fluctuation shape function of the 2-nd kind, $F S_{\delta}^{2}(\Pi, \Delta)$, if it depends on $l$ as a parameter and the conditions hold

(i) $\partial^{k} g \in O\left(l^{\alpha-k}\right)$ for $k=0,1, \ldots, \alpha, \alpha=2, \partial^{0} g \equiv g$

(ii) $\langle g\rangle(\mathbf{x}) \approx 0 \forall \mathbf{x} \in \Pi_{\Delta}$

where $l$ is the microstructure parameter. Condition $(3.7)_{(i i)}$ can be replaced by $\langle\mu g\rangle(\mathbf{x}) \approx 0$ for every $\mathbf{x} \in \Pi_{\Delta}$, where $\mu>0$ is a certain tolerance-periodic function. 


\subsection{Fundamental assumptions of the tolerance modelling}

The tolerance modelling is based on two fundamental modelling assumptions which are formulated in general form in the books by Woźniak et al. $(2008,2010)$. Here, they are shown below in the form for thin periodic plates.

The micro-macro decomposition is the first assumption in which it is assumed that the deflection and the in-plane displacements can be decomposed as

$$
\begin{array}{ll}
w(\mathbf{x}, t)=W(\mathbf{x}, t)+g^{A}(\mathbf{x}) V^{A}(\mathbf{x}, t) & A=1, \ldots, N \\
u_{\alpha}^{0}(\mathbf{x}, t)=U_{\alpha}(\mathbf{x}, t)+f^{a}(\mathbf{x}) T_{\alpha}^{a}(\mathbf{x}, t) & a=1, \ldots, m
\end{array}
$$

and the functions $W(\cdot, t), V^{A}(\cdot, t) \in S V_{\delta}^{2}(\Pi, \Delta), U_{\alpha}(\cdot, t), T_{\alpha}^{a}(\cdot, t) \in S V_{\delta}^{1}(\Pi, \Delta)$ are the basic unknowns; $g^{A}(\cdot) \in F S_{\delta}^{2}(\Pi, \Delta), f^{a}(\cdot) \in F S_{\delta}^{1}(\Pi, \Delta)$ are the known fluctuation shape functions. The functions $W(\cdot, t)$ and $U_{\alpha}(\cdot, t)$ are called the macrodeflection and the in-plane macrodisplacements, respectively; $V^{A}(\cdot, t)$ and $T_{\alpha}^{a}(\cdot, t)$ are called the fluctuation amplitudes of the deflection and the in-plane displacements, respectively. The fluctuation shape function can be obtained as solutions to eigenvalue problems posed on the periodicity cell, cf. Jędrysiak (2009). However, in most cases, they are assumed in an approximate form as: trigonometric functions $\left(g^{A}\right)$ or saw-type functions $\left(f^{a}\right)$, see Jędrysiak $(2003,2013)$.

Moreover, similar assumptions to (3.8) are introduced for virtual displacements $\bar{w}(\cdot), \bar{u}_{\alpha}^{0}(\cdot)$

$$
\begin{array}{ll}
\bar{w}(\mathbf{x})=\bar{W}(\mathbf{x})+g^{A}(\mathbf{x}) \bar{V}^{A}(\mathbf{x}) & A=1, \ldots, N \\
\bar{u}_{\alpha}^{0}(\mathbf{x})=\bar{U}_{\alpha}(\mathbf{x})+f^{a}(\mathbf{x}) \bar{T}_{\alpha}^{a}(\mathbf{x}) & a=1, \ldots, m
\end{array}
$$

with slowly-varying functions $\bar{W}(\cdot), \bar{V}^{A}(\cdot) \in S V_{\delta}^{2}(\Pi, \Delta), \bar{U}_{\alpha}(\cdot), \bar{T}_{\alpha}^{a}(\cdot) \in S V_{\delta}^{1}(\Pi, \Delta)$.

In the tolerance averaging approximation, the terms $O(\delta)$ are assumed to be negligibly small in the course of modelling, i.e. they can be omitted in the following formulas

(i) $\quad\langle\varphi\rangle(\mathbf{x})=\langle\widetilde{\varphi}(\mathbf{x})+O(\delta)$

(ii) $\quad\langle\varphi\rangle F\rangle(\mathbf{x})=\langle\varphi\rangle(\mathbf{x}) F(\mathbf{x})+O(\delta)$

(iii) $\langle\varphi\rangle(g F)_{, \gamma}(\mathbf{x})=\langle\varphi\rangle g_{, \gamma}(\mathbf{x}) F(\mathbf{x})+O(\delta)$

$\mathbf{x} \in \Pi ; \quad \gamma=1, \alpha ; \quad \alpha=1,2 ; \quad 0<\delta \ll 1 ;$

$\varphi \in T P_{\delta}^{\alpha}(\Pi, \Delta) ; \quad F \in S V_{\delta}^{\alpha}(\Pi, \Delta) ; \quad g \in F S_{\delta}^{\alpha}(\Pi, \Delta)$

\subsection{The modelling procedure}

The above concepts and fundamental assumptions are used in the modelling procedure. This procedure can be divided into four steps.

In the first step, micro-macro decompositions (3.8) and (3.9) are substituted into virtual work equation (2.6) of such a plate resting on a foundation. Then, in the second step, the averaging operation is used to average the resulting equation over the periodicity cell, see Jędrysiak (2003). 
In the next step, we arrive at the tolerance averaged virtual work equation after using formulas (3.10) of the tolerance averaging approximation (Jędrysiak, 2003). Applying the following denotations of some averaged parameters, being averaged constitutive relations

$$
\begin{array}{rlrl}
M_{\alpha \beta} & \equiv-\left\langle\int_{-d / 2}^{d / 2} s_{\alpha \beta} z d z\right\rangle & M^{A} \equiv-\left\langle g_{, \alpha \beta}^{A} \int_{-d / 2}^{d / 2} s_{\alpha \beta} z d z\right\rangle \\
N_{\alpha \beta}=\left\langle\int_{-d / 2}^{d / 2} s_{\alpha \beta} d z\right\rangle & N_{\alpha}^{a} \equiv\left\langle\int_{-d / 2}^{d / 2} s_{\alpha \beta} f_{, \beta}^{a} d z\right\rangle \\
Q_{\alpha}^{A} \equiv\left\langle\int_{-d / 2}^{d / 2} s_{\alpha \beta} g_{, \beta}^{A} d z\right\rangle & R^{A B} \equiv\left\langle\int_{-d / 2}^{d / 2} s_{\alpha \beta} g_{, \alpha}^{A} g_{, \beta}^{B} d z\right\rangle
\end{array}
$$

this tolerance averaged virtual work equation can be written as

$$
\begin{aligned}
\int_{\Pi} & \left.\langle\mu\rangle \ddot{W}+\left\langle\mu g^{B}\right\rangle \ddot{V}^{B}\right) \delta W d a+\int_{\Pi}\left(\left\langle\mu g^{A}\right\rangle \ddot{W}+\left\langle\mu g^{A} g^{B}\right\rangle \ddot{V}^{B}\right) \delta V^{A} d a \\
& +\int_{\Pi}\left(\langle\mu\rangle \ddot{U}_{\alpha}+\left\langle\mu f^{b}\right\rangle \ddot{T}_{\alpha}^{b}-\langle i\rangle \ddot{W}_{, \alpha}-\left\langle i g_{, \alpha}^{B}\right\rangle \ddot{V}^{B}\right) \delta U_{\alpha} d a \\
& +\int_{\Pi}\left(\left\langle\mu f^{a}\right\rangle \ddot{U}_{\alpha}+\left\langle\mu f^{a} f^{b}\right\rangle \ddot{T}_{\alpha}^{b}-\left\langle i f^{a}\right\rangle \ddot{W}_{, \alpha}-\left\langle i f^{a} g_{, \alpha}^{B}\right\rangle \ddot{V}^{B}\right) \delta T_{\alpha}^{a} d a \\
& -\int_{\Pi}\left(-\langle i\rangle \ddot{U}_{\alpha, \alpha}-\left\langle i f^{b}\right\rangle \ddot{T}_{\alpha, \alpha}^{b}+\langle j\rangle \ddot{W}_{, \alpha \alpha}+\left\langle j g_{, \alpha}^{B}\right\rangle \ddot{V}_{, \alpha}^{B}\right) \delta W d a \\
& +\int_{\Pi}\left(-\left\langle i g_{, \alpha}^{A}\right\rangle \ddot{U}_{\alpha}-\left\langle i f^{b} g_{, \alpha}^{A}\right\rangle \ddot{T}_{\alpha}^{b}+\left\langle j g_{, \alpha}^{A}\right\rangle \ddot{W}_{, \alpha}+\left\langle j g_{, \alpha}^{A} g_{, \alpha}^{B}\right\rangle \ddot{V}^{B}\right) \delta V^{A} d a \\
& -\int_{\Pi} N_{\alpha \beta, \beta} \delta U_{\alpha} d a+\int_{\Pi} N_{\alpha}^{a} \delta T_{\alpha}^{a} d a \\
& +\int_{\Pi}\left[M_{\alpha \beta, \alpha \beta}-\left(N_{\alpha \beta} W_{, \alpha}+Q_{\beta}^{A} V^{A}\right)_{, \beta}\right] \delta W d a \\
& +\int_{\Pi}\left(M^{A}+Q_{\alpha}^{A} W_{, \alpha}+R^{A B} V^{B}\right) \delta V^{A} d a \\
& =\int_{\Pi} p \delta W d a-\int_{\Pi}\left(\langle k\rangle W+\left\langle k g^{B}\right\rangle V^{B}\right) \delta W d a \\
& -\int_{\Pi}\left(\left\langle k g^{A}\right\rangle W+\left\langle k g^{A} g^{B}\right\rangle V^{B}\right) \delta V^{A} d a \\
& -\int_{\Pi}\left(\langle\widehat{\mu}\rangle \ddot{W}+\left\langle\widehat{\mu} g^{B}\right\rangle \ddot{V}^{B}\right) \delta W d a-\int_{\Pi}\left(\left\langle\widehat{\mu} g^{A}\right\rangle \ddot{W}+\left\langle\widehat{\mu} g^{A} g^{B}\right\rangle \ddot{V}^{B}\right) \delta V^{A} d a \\
& -\int_{\Pi}\left(\langle c\rangle \dot{W}+\left\langle c g^{B}\right\rangle \dot{V}^{B}\right) \delta W d a-\int_{\Pi}\left(\left\langle c g^{A}\right\rangle \dot{W}+\left\langle c g^{A} g^{B}\right\rangle \dot{V}^{B}\right) \delta V^{A} d a \\
&
\end{aligned}
$$

Then, using the divergence theorem and the du Bois-Reymond lemma to equation (3.12), after some manipulations, governing equations of the proposed approximate tolerance model can be obtained. 


\section{Governing equations}

\subsection{Tolerance model equations}

Let us introduce denotations

$$
\begin{aligned}
& B_{\alpha \beta \gamma \delta} \equiv\left\langle b_{\alpha \beta \gamma \delta}\right\rangle \\
& B_{\alpha \gamma}^{a b} \equiv\left\langle b_{\alpha \beta \gamma \delta} f_{, \beta}^{a} f_{, \delta}^{b}\right\rangle \\
& D_{\alpha \beta}^{A} \equiv\left\langle d_{\alpha \beta \gamma \delta} g_{, \gamma \delta}^{A}\right\rangle \\
& F_{\alpha}^{A B C} \equiv l^{-3}\left\langle b_{\alpha \beta \gamma \delta} g_{, \beta}^{A} g_{, \gamma}^{B} g_{, \delta}^{C}\right\rangle \\
& F_{\alpha \beta}^{A B} \equiv l^{-2}\left\langle b_{\alpha \beta \gamma \delta} g_{, \gamma}^{A} g_{, \delta}^{B}\right\rangle \\
& F_{\alpha}^{a B C} \equiv l^{-2}\left\langle b_{\alpha \beta \gamma \delta} f_{, \beta}^{a} g_{, \gamma}^{B} g_{, \delta}^{C}\right\rangle \\
& G_{\alpha \beta \gamma}^{A} \equiv l^{-1}\left\langle h_{\alpha \beta \gamma \delta} g_{, \delta}^{A}\right\rangle \\
& G_{\gamma}^{A B} \equiv l^{-1}\left\langle h_{\alpha \beta \gamma \delta} g_{, \alpha \beta}^{A} g_{, \delta}^{B}\right\rangle \\
& H_{\alpha \beta \gamma \delta} \equiv\left\langle h_{\alpha \beta \gamma \delta}\right\rangle \\
& H_{\alpha \gamma \delta}^{a} \equiv\left\langle h_{\alpha \beta \gamma \delta} f_{, \beta}^{a}\right\rangle \\
& \widetilde{B}_{\alpha \beta \gamma \delta} \equiv\left\langle\widetilde{b}_{\alpha \beta \gamma \delta}\right\rangle \\
& \widetilde{B}_{\alpha \gamma}^{a b} \equiv\left\langle\widetilde{b}_{\alpha \beta \gamma \delta} f_{, \beta}^{a} f_{, \delta}^{b}\right\rangle \\
& \widetilde{D}_{\alpha \beta}^{A} \equiv\left\langle\widetilde{d}_{\alpha \beta \gamma \delta} g_{, \gamma \delta}^{A}\right\rangle \\
& \widetilde{F}_{\alpha}^{A B C} \equiv l^{-3}\left\langle\widetilde{b}_{\alpha \beta \gamma \delta} g_{, \beta}^{A} g_{, \gamma}^{B} g_{, \delta}^{C}\right\rangle \\
& \widetilde{F}_{\alpha \beta}^{A B} \equiv l^{-2}\left\langle\widetilde{b}_{\alpha \beta \gamma \delta} g_{, \gamma}^{A} g_{, \delta}^{B}\right\rangle \\
& \widetilde{F}_{\alpha}^{a B C} \equiv l^{-2}\left\langle\widetilde{b}_{\alpha \beta \gamma \delta} f_{, \beta}^{A} g_{, \gamma}^{B} g_{, \delta}^{C}\right\rangle \\
& \widetilde{G}_{\alpha \beta \gamma}^{A} \equiv l^{-1}\left\langle\widetilde{h}_{\alpha \beta \gamma \delta} g_{, \delta}^{A}\right\rangle \\
& \widetilde{G}_{\gamma}^{A B} \equiv l^{-1}\left\langle\widetilde{h}_{\alpha \beta \gamma \delta} g_{, \alpha \beta}^{A} g_{, \delta}^{B}\right\rangle \\
& \widetilde{H}_{\alpha \beta \gamma \delta} \equiv\left\langle\widetilde{h}_{\alpha \beta \gamma \delta}\right\rangle \\
& \widetilde{H}_{\alpha \gamma \delta}^{a} \equiv\left\langle\widetilde{h}_{\alpha \beta \gamma \delta} f_{, \beta}^{a}\right\rangle \\
& m \equiv\langle\mu\rangle \\
& m^{A B} \equiv l^{-4}\left\langle\mu g^{A} g^{B}\right\rangle \\
& \vartheta_{\alpha}^{A} \equiv l^{-1}\left\langle j g_{, \alpha}^{A}\right\rangle \\
& \theta \equiv\langle i\rangle \\
& \theta_{\alpha}^{A} \equiv l^{-1}\left\langle i g_{, \alpha}^{A}\right\rangle \\
& C \equiv\langle c\rangle \\
& C^{A B} \equiv l^{-4}\left\langle c g^{A} g^{B}\right\rangle \\
& K^{A} \equiv l^{-2}\left\langle k g^{A}\right\rangle \\
& \widehat{m} \equiv\langle\widehat{\mu}\rangle \\
& \widehat{m}^{A B} \equiv l^{-4}\left\langle\widehat{\mu} g^{A} g^{B}\right\rangle \\
& \bar{m}^{a b} \equiv l^{-4}\left\langle\mu f^{a} f^{b}\right\rangle \\
& B_{\alpha \beta \gamma}^{a} \equiv\left\langle b_{\alpha \beta \gamma \delta} f_{, \delta}^{a}\right\rangle \\
& D_{\alpha \beta \gamma \delta} \equiv\left\langle d_{\alpha \beta \gamma \delta}\right\rangle \\
& D^{A B} \equiv\left\langle d_{\alpha \beta \gamma \delta} g_{, \alpha \beta}^{A} g_{, \gamma \delta}^{B}\right\rangle \\
& F_{\alpha \beta \gamma}^{A} \equiv l^{-1}\left\langle b_{\alpha \beta \gamma \delta} g_{, \delta}^{A}\right\rangle \\
& F_{\alpha \gamma}^{a B} \equiv l^{-1}\left\langle b_{\alpha \beta \gamma \delta} f_{, \beta}^{a} g_{, \delta}^{B}\right\rangle \\
& F^{A B C D} \equiv l^{-4}\left\langle b_{\alpha \beta \gamma \delta} g_{, \alpha}^{A} g_{, \beta}^{B} g_{, \gamma}^{C} g_{, \delta}^{D}\right\rangle \\
& G_{\alpha \beta}^{A B} \equiv l^{-2}\left\langle h_{\alpha \beta \gamma \delta} g_{, \gamma}^{A} g_{, \delta}^{B}\right\rangle \\
& G^{A B C} \equiv l^{-2}\left\langle h_{\alpha \beta \gamma \delta} g_{, \alpha \beta}^{A} g_{, \gamma}^{B} g_{, \delta}^{C}\right\rangle \\
& H_{\alpha \beta}^{A} \equiv\left\langle h_{\alpha \beta \gamma \delta} g_{, \gamma \delta}^{A}\right\rangle \\
& H_{\alpha}^{a B} \equiv\left\langle h_{\alpha \beta \gamma \delta} f_{, \beta}^{a} g_{, \gamma \delta}^{B}\right\rangle \\
& \widetilde{B}_{\alpha \beta \gamma}^{a} \equiv\left\langle\widetilde{b}_{\alpha \beta \gamma \delta} f_{, \delta}^{a}\right\rangle \\
& \widetilde{D}_{\alpha \beta \gamma \delta} \equiv\left\langle\widetilde{d}_{\alpha \beta \gamma \delta}\right\rangle \\
& \widetilde{D}^{A B} \equiv\left\langle\widetilde{d}_{\alpha \beta \gamma \delta} g_{, \alpha \beta}^{A} g_{, \gamma \delta}^{B}\right\rangle \\
& \widetilde{F}_{\alpha \beta \gamma}^{A} \equiv l^{-1}\left\langle\widetilde{b}_{\alpha \beta \gamma \delta} g_{, \delta}^{A}\right\rangle \\
& \widetilde{F}_{\alpha \gamma}^{a B} \equiv l^{-1}\left\langle\widetilde{b}_{\alpha \beta \gamma \delta} f_{, \beta}^{a} g_{, \delta}^{B}\right\rangle \\
& \widetilde{F}^{A B C D} \equiv l^{-4}\left\langle\widetilde{b}_{\alpha \beta \gamma \delta} g_{, \alpha}^{A} g_{, \beta}^{B} g_{, \gamma}^{C} g_{, \delta}^{D}\right\rangle \\
& \widetilde{G}_{\alpha \beta}^{A B} \equiv l^{-2}\left\langle\widetilde{h}_{\alpha \beta \gamma \delta} g_{, \gamma}^{A} g_{, \delta}^{B}\right\rangle \\
& \widetilde{G}^{A B C} \equiv l^{-2}\left\langle\widetilde{h}_{\alpha \beta \gamma \delta} g_{, \alpha \beta}^{A} g_{, \gamma}^{B} g_{, \delta}^{C}\right\rangle \\
& \widetilde{H}_{\alpha \beta}^{A} \equiv\left\langle\widetilde{h}_{\alpha \beta \gamma \delta} g_{, \gamma \delta}^{A}\right\rangle \\
& \widetilde{H}_{\alpha}^{a B} \equiv\left\langle\widetilde{h}_{\alpha \beta \gamma \delta} f_{, \beta}^{a} g_{, \gamma \delta}^{B}\right\rangle \\
& m^{A} \equiv l^{-2}\left\langle\mu g^{A}\right\rangle \\
& \vartheta \equiv\langle j\rangle \\
& \vartheta_{\alpha \beta}^{A B} \equiv l^{-2}\left\langle j g_{, \alpha}^{A} g_{, \beta}^{B}\right\rangle \\
& \theta^{a} \equiv l^{-1}\left\langle i f^{a}\right\rangle \\
& \theta_{\alpha}^{a A} \equiv l^{-2}\left\langle i f^{a} g_{, \alpha}^{A}\right\rangle \\
& C^{A} \equiv l^{-2}\left\langle c g^{A}\right\rangle \\
& K \equiv\langle k\rangle \\
& K^{A B} \equiv l^{-4}\left\langle k g^{A} g^{B}\right\rangle \\
& \widehat{m}^{A} \equiv l^{-2}\left\langle\widehat{\mu} g^{A}\right\rangle \\
& \bar{m}^{a} \equiv l^{-1}\left\langle\mu f^{a}\right\rangle \\
& P \equiv\langle p\rangle \quad P^{A} \equiv l^{-2}\left\langle p g^{A}\right\rangle
\end{aligned}
$$

Using the tolerance modelling procedure, a system of equations for the in-plane macrodisplacements $U_{\alpha}$, fluctuation amplitudes of the in-plane macrodisplacements $T_{\alpha}^{a}$, macrodeflection $W$, fluctuation amplitudes of the deflection $V^{A}$ can be derived: 
- constitutive equations

$$
\begin{aligned}
& M_{\alpha \beta}=-H_{\alpha \beta \gamma \delta}\left(U_{\gamma, \delta}+\frac{1}{2} W_{, \gamma} W_{, \delta}\right)-H_{\alpha \beta \gamma}^{a} T_{\gamma}^{a}+D_{\alpha \beta \gamma \delta} W_{, \gamma \delta}+D_{\alpha \beta}^{A} V^{A} \\
& -l G_{\alpha \beta \gamma}^{A}\left[W_{, \gamma} V^{A}+\left(\dot{W}_{, \gamma} V^{A}+W_{, \gamma} \dot{V}^{A}\right)\right]-\frac{1}{2} l^{2} G_{\alpha \beta}^{A B}\left[V^{A} V^{B}+\left(\dot{V}^{A} V^{B}+V^{A} \dot{V}^{B}\right)\right] \\
& -\widetilde{H}_{\alpha \beta \gamma \delta}\left[\dot{U}_{\gamma, \delta}-\frac{1}{2}\left(\dot{W}_{, \gamma} W_{, \delta}+W_{, \gamma} \dot{W}_{, \delta}\right)\right]-\widetilde{H}_{\alpha \beta \gamma}^{a} \dot{T}_{\gamma}^{a}+\widetilde{D}_{\alpha \beta \gamma \delta} \dot{W}_{, \gamma \delta}+\widetilde{D}_{\alpha \beta}^{A} \dot{V}^{A} \\
& M^{A}=-H_{\alpha \beta}^{A}\left(U_{\gamma, \delta}+\frac{1}{2} W_{, \gamma} W_{, \delta}\right)-H_{\alpha}^{a A} T_{\alpha}^{a}+D_{\alpha \beta}^{A} W_{, \gamma \delta}+D^{A B} V^{B} \\
& -l G_{\gamma}^{A B} W_{, \gamma} V^{B}-\frac{1}{2} l^{2} G^{A B C} V^{B} V^{C}-\widetilde{H}_{\alpha \beta}^{A}\left[\dot{U}_{\gamma, \delta}+\frac{1}{2}\left(\dot{W}_{, \gamma} W_{, \delta}+W_{, \gamma} \dot{W}_{, \delta}\right)\right] \\
& -\widetilde{H}_{\alpha}^{a A} \dot{T}_{\alpha}^{a}+\widetilde{D}_{\alpha \beta}^{A} \dot{W}_{, \gamma \delta}+\widetilde{D}^{A B} \dot{V}^{B}-l \widetilde{G}_{\gamma}^{A B}\left(\dot{W}_{, \gamma} V^{B}+W_{, \gamma} \dot{V}^{B}\right) \\
& -\frac{1}{2} l^{2} \widetilde{G}^{A B C}\left(\dot{V}^{B} V^{C}+V^{B} \dot{V}^{C}\right) \\
& N_{\alpha \beta}=B_{\alpha \beta \gamma \delta}\left(U_{\gamma, \delta}+\frac{1}{2} W_{, \gamma} W_{, \delta}\right)+B_{\alpha \beta \gamma}^{a} T_{\gamma}^{a}-H_{\alpha \beta \gamma \delta} W_{, \gamma \delta}-H_{\alpha \beta}^{A} V^{A}+l F_{\alpha \beta \gamma}^{A} W_{, \gamma} V^{A} \\
& +\frac{1}{2} l^{2} F_{\alpha \beta}^{A B} V^{A} V^{B}+\widetilde{B}_{\alpha \beta \gamma \delta}\left[\dot{U}_{\gamma, \delta}+\frac{1}{2}\left(\dot{W}_{, \gamma} W_{, \delta}+W_{, \gamma} \dot{W}_{, \delta}\right)\right]+\widetilde{B}_{\alpha \beta \gamma}^{a} \dot{T}_{\gamma}^{a}-\widetilde{H}_{\alpha \beta \gamma \delta} \dot{W}_{, \gamma \delta} \\
& -\widetilde{H}_{\alpha \beta}^{A} \dot{V}^{A}+l \widetilde{F}_{\alpha \beta \gamma}^{A}\left(\dot{W}_{, \gamma} V^{A}+W_{, \gamma} \dot{V}^{A}\right)+\frac{1}{2} l^{2} \widetilde{F}_{\alpha \beta}^{A B}\left(\dot{V}^{A} V^{B}+V^{A} \dot{V}^{B}\right) \\
& N_{\alpha}^{a}=B_{\alpha \gamma \delta}^{a}\left(U_{\gamma, \delta}+\frac{1}{2} W_{, \gamma} W_{, \delta}\right)+B_{\alpha \gamma}^{a b} T_{\gamma}^{b}-H_{\alpha \gamma \delta}^{a} W_{, \gamma \delta}-H_{\alpha}^{a B} V^{B}+l F_{\alpha \gamma}^{a B} W_{, \gamma} V^{B} \\
& +\frac{1}{2} l^{2} F_{\alpha}^{a B C} V^{B} V^{C}+\widetilde{B}_{\alpha \gamma \delta}^{a}\left[\dot{U}_{\gamma, \delta}+\frac{1}{2}\left(\dot{W}_{, \gamma} W_{, \delta}+W_{, \gamma} \dot{W}_{, \delta}\right)\right]+\widetilde{B}_{\alpha \gamma}^{a b} \dot{T}_{\gamma}^{b}-\widetilde{H}_{\alpha \gamma \delta}^{a} \dot{W}_{, \gamma \delta} \\
& -\widetilde{H}_{\alpha}^{a B} \dot{V}^{B}+l \widetilde{F}_{\alpha \gamma}^{a B}\left(\dot{W}_{, \gamma} V^{B}+W_{, \gamma} \dot{V}^{B}\right)+\frac{1}{2} l^{2} \widetilde{F}_{\alpha}^{a B C}\left(\dot{V}^{B} V^{C}+V^{B} \dot{V}^{C}\right) \\
& Q_{\alpha}^{A}=l F_{\alpha \gamma \delta}^{A}\left(U_{\gamma, \delta}+\frac{1}{2} W_{, \gamma} W_{, \delta}\right)+l F_{\alpha \gamma}^{a A} T_{\gamma}^{a}-l G_{\alpha \gamma \delta}^{A} W_{, \gamma \delta}-l G_{\alpha}^{A B} V^{B}+l^{2} F_{\alpha \gamma}^{A B} W_{, \gamma} V^{B} \\
& +\frac{1}{2} l^{3} F_{\alpha}^{A B C} V^{B} V^{C}+l \widetilde{F}_{\alpha \gamma \delta}^{A} \dot{U}_{\gamma, \delta}+l \widetilde{F}_{\alpha \gamma}^{a A}\left[\dot{T}_{\gamma}^{a}+\frac{1}{2}\left(\dot{W}_{, \gamma} W_{, \delta}+W_{, \gamma} \dot{W}_{, \delta}\right)\right] \\
& -l \widetilde{G}_{\alpha \gamma \delta}^{A} \dot{W}_{, \gamma \delta}-l \widetilde{G}_{\alpha}^{A B} \dot{V}^{B}+l^{2} \widetilde{F}_{\alpha \gamma}^{A B}\left(\dot{W}_{, \gamma} V^{B}+W_{, \gamma} \dot{V}^{B}\right)+\frac{1}{2} l^{3} \widetilde{F}_{\alpha}^{A B C}\left(\dot{V}^{B} V^{C}+V^{B} \dot{V}^{C}\right) \\
& R^{A B}=l^{2} F_{\gamma \delta}^{A B}\left(U_{\gamma, \delta}+\frac{1}{2} W_{, \gamma} W_{, \delta}\right)+l^{2} F_{\gamma}^{a A B} T_{\gamma}^{a}-l^{2} G_{\gamma \delta}^{A B} W_{, \gamma \delta}-l^{2} G^{A B C} V^{C}+l^{3} F_{\gamma}^{A B C} W_{, \gamma} V^{C} \\
& +\frac{1}{2} l^{4} F^{A B C D} V^{C} V^{D}+l^{2} \widetilde{F}_{\gamma \delta}^{A B}\left[\dot{U}_{\gamma, \delta}+\frac{1}{2}\left(\dot{W}_{, \gamma} W_{, \delta}+W_{, \gamma} \dot{W}_{, \delta}\right)\right]+l^{2} \widetilde{F}_{\gamma}^{a A B} \dot{T}_{\gamma}^{a}-l^{2} \widetilde{G}
\end{aligned}
$$

- equilibrium equations

$$
\begin{aligned}
& M_{\alpha \beta, \alpha \beta}-\left(N_{\alpha \beta} W_{, \alpha}+Q_{\beta}^{A} V^{A}\right)_{, \beta}+(m+\widehat{m}) \ddot{W}+l^{2}\left(m^{A}+\widehat{m}^{A}\right) \ddot{V}^{A}-\vartheta \ddot{W}_{, \alpha \alpha}-l \vartheta_{\alpha}^{A} \ddot{V}_{, \alpha}^{A} \\
& \quad+K W+l^{2} K^{A} V^{A}+C \dot{W}+l^{2} C^{A} \dot{V}^{A}+\theta \ddot{U}_{\alpha, \alpha}+l \theta^{a} \ddot{T}_{\alpha, \alpha}^{a}=P \\
& M^{A}+Q_{\alpha}^{A} W_{, \alpha}+R^{A B} V^{B}+l^{2}\left(m^{A}+\widehat{m}^{A}\right) \ddot{W}+l \vartheta_{\alpha}^{A} \ddot{W}_{, \alpha}+l^{2}\left(l^{2} m^{A B}+l^{2} \widehat{m}^{A B}+\vartheta_{\alpha \beta}^{A B}\right) \ddot{V}^{B} \\
& \quad+l^{2} K^{A} W+l^{4} K^{A B} V^{B}+l^{2} C^{A} \dot{W}+l^{4} C^{A B} \dot{V}^{B}-l \theta_{\alpha}^{A} \ddot{U}_{\alpha}-l^{2} \theta_{\alpha}^{a A} \ddot{T}_{\alpha}^{a}=l^{2} P^{A} \\
& -N_{\alpha \beta, \beta}+m \ddot{U}_{\alpha}+l \bar{m}^{a} \ddot{T}_{\alpha}^{a}-\theta \ddot{W}_{, \alpha}-l \theta_{\alpha}^{A} \ddot{V}^{A}=0 \\
& N_{\alpha}^{a}+l \bar{m}^{a} \ddot{U}_{\alpha}+l^{2} \bar{m}^{a b} \ddot{T}_{\alpha}^{b}-l \theta^{a} \ddot{W}_{, \alpha}-l^{2} \theta_{\alpha}^{a A} \ddot{V}^{A}=0
\end{aligned}
$$

Equations (4.2) and (4.3) together with micro-macro decompositions (3.8) constitute the nonlinear tolerance model of thin visco-elastic periodic plates resting on a foundation with damping if the plate properties are not even functions of $z$. This model describes the effect of the microstructure size on the overall plate behaviour by terms with the microstructure 
parameter $l$. For the considered plates, boundary conditions have to be formulated only for the macrodeflection $W$ and the in-plane macrodisplacements $U_{\alpha}$. Moreover, the basic unknowns of equations (4.2) and (4.3) have to satisfy the following conditions: $W(\cdot, t), V^{A}(\cdot, t) \in S V_{\delta}^{2}(\Pi, \Delta)$, $U_{\alpha}(\cdot, t), T_{\alpha}^{a}(\cdot, t) \in S V_{\delta}^{1}(\Pi, \Delta)$, i.e. they are slowly-varying functions in $x$.

In the next considerations, it is assumed that the plate properties are even functions of $z$, i.e. plates under consideration have the symmetry plane $z=0$. Hence, some coefficients (4.1) are equal to zero

$$
\begin{aligned}
& H_{\alpha \beta \gamma \delta}=H_{\alpha \beta}^{A}=H_{\alpha \gamma \delta}^{a}=H_{\alpha}^{a B}=G_{\alpha \beta \gamma}^{A}=G_{\alpha \beta}^{A B}=G_{\gamma}^{A B}=G^{A B C}=0 \\
& \widetilde{H}_{\alpha \beta \gamma \delta}=\widetilde{H}_{\alpha \beta}^{A}=\widetilde{H}_{\alpha \gamma \delta}^{a}=\widetilde{H}_{\alpha}^{a B}=\widetilde{G}_{\alpha \beta \gamma}^{A}=\widetilde{G}_{\alpha \beta}^{A B}=\widetilde{G}_{\gamma}^{A B}=\widetilde{G}^{A B C}=0 \\
& \theta=\theta^{a}=\theta_{\alpha}^{A}=\theta_{\alpha}^{a A}=0
\end{aligned}
$$

Equations (4.2) and (4.3) take the following form:

- constitutive equations

$$
\begin{aligned}
M_{\alpha \beta} & =D_{\alpha \beta \gamma \delta} W_{, \gamma \delta}+D_{\alpha \beta}^{A} V^{A}+\widetilde{D}_{\alpha \beta \gamma \delta} \dot{W}_{, \gamma \delta}+\widetilde{D}_{\alpha \beta}^{A} \dot{V}^{A} \\
M^{A} & =D_{\alpha \beta}^{A} W_{, \gamma \delta}+D^{A B} V^{B}+\widetilde{D}_{\alpha \beta}^{A} \dot{W}_{, \gamma \delta}+\widetilde{D}^{A B} \dot{V}^{B} \\
N_{\alpha \beta} & =B_{\alpha \beta \gamma \delta}\left(U_{\gamma, \delta}+\frac{1}{2} W_{, \gamma} W_{, \delta}\right)+B_{\alpha \beta \gamma}^{a} T_{\gamma}^{a}+l F_{\alpha \beta \gamma}^{A} W_{, \gamma} V^{A}+\frac{1}{2} l^{2} F_{\alpha \beta}^{A B} V^{A} V^{B} \\
& +\widetilde{B}_{\alpha \beta \gamma \delta}\left[\dot{U}_{\gamma, \delta}+\frac{1}{2}\left(\dot{W}_{, \gamma} W_{, \delta}+W_{, \gamma} \dot{W}_{, \delta}\right)\right]+\widetilde{B}_{\alpha \beta \gamma}^{a} \dot{T}_{\gamma}^{a} \\
& +l \widetilde{F}_{\alpha \beta \gamma}^{A}\left(\dot{W}_{, \gamma} V^{A}+W_{, \gamma} \dot{V}^{A}\right)+\frac{1}{2} l^{2} \widetilde{F}_{\alpha \beta}^{A B}\left(\dot{V}^{A} V^{B}+V^{A} \dot{V}^{B}\right) \\
N_{\alpha}^{a}= & B_{\alpha \gamma \delta}^{a}\left(U_{\gamma, \delta}+\frac{1}{2} W_{, \gamma} W_{, \delta}\right)+B_{\alpha \gamma}^{a b} T_{\gamma}^{b}+l F_{\alpha \gamma}^{a B} W_{, \gamma} V^{B}+\frac{1}{2} l^{2} F_{\alpha}^{a B C} V^{B} V^{C} \\
& +\widetilde{B}_{\alpha \gamma \delta}^{a}\left[\dot{U}_{\gamma, \delta}+\frac{1}{2}\left(\dot{W}_{, \gamma} W_{, \delta}+W_{, \gamma} \dot{W}_{, \delta}\right)\right]+\widetilde{B}_{\alpha \gamma}^{a b} \dot{T}_{\gamma}^{b} \\
& +l \widetilde{F}_{\alpha \gamma}^{a B}\left(\dot{W}_{, \gamma} V^{B}+W_{, \gamma} \dot{V}^{B}\right)+\frac{1}{2} l^{2} \widetilde{F}_{\alpha}^{a B C}\left(\dot{V}^{B} V^{C}+V^{B} \dot{V}^{C}\right) \\
Q_{\alpha}^{A} & =l F_{\alpha \gamma \delta}^{A}\left(U_{\gamma, \delta}+\frac{1}{2} W_{, \gamma} W_{, \delta}\right)+l F_{\alpha \gamma}^{a A} T_{\gamma}^{a}+l^{2} F_{\alpha \gamma}^{A B} W_{, \gamma} V^{B}+\frac{1}{2} l^{3} F_{\alpha}^{A B C} V^{B} V^{C} \\
& +l \widetilde{F}_{\alpha \gamma \delta}^{A} \dot{U}_{\gamma, \delta}+l \widetilde{F}_{\alpha \gamma}^{a A}\left[\dot{T}_{\gamma}^{a}+\frac{1}{2}\left(\dot{W}_{, \gamma} W_{, \delta}+W_{, \gamma} \dot{W}_{, \delta}\right)\right]+l^{2} \widetilde{F}_{\alpha \gamma}^{A B}\left(\dot{W}_{, \gamma} V^{B}+W_{, \gamma} \dot{V}^{B}\right) \\
& +\frac{1}{2} l^{3} \widetilde{F}_{\alpha}^{A B C}\left(\dot{V}^{B} V^{C}+V^{B} \dot{V}^{C}\right) \\
R^{A B} & =l^{2} F_{\gamma \delta}^{A B}\left(U_{\gamma, \delta}+\frac{1}{2} W_{, \gamma} W_{, \delta}\right)+l^{2} F_{\gamma}^{a A B} T_{\gamma}^{a}+l^{3} F_{\gamma}^{A B C} W_{, \gamma} V^{C}+\frac{1}{2} l^{4} F^{A B C D} V^{C} V^{D} \\
& +l^{2} \widetilde{F}_{\gamma}^{a A B} \dot{T}_{\gamma}^{a}+l^{2} \widetilde{F}_{\gamma \delta}^{A B}\left[\dot{U}_{\gamma, \delta}+\frac{1}{2}\left(\dot{W}_{, \gamma} W_{, \delta}+W_{, \gamma} \dot{W}_{, \delta}\right)\right] \\
& +l^{3} \widetilde{F}_{\gamma}^{A B C}\left(\dot{W}_{, \gamma} V^{C}+W_{, \gamma} \dot{V}^{C}\right)+\frac{1}{2} l^{4} \widetilde{F}^{A B C D}\left(\dot{V}^{C} V^{D}+V^{C} \dot{V}^{D}\right)
\end{aligned}
$$

— equilibrium equations

$$
\begin{aligned}
& M_{\alpha \beta, \alpha \beta}-\left(N_{\alpha \beta} W_{, \alpha}+Q_{\beta}^{A} V^{A}\right)_{, \beta}+(m+\widehat{m}) \ddot{W}+l^{2}\left(m^{A}+\widehat{m}^{A}\right) \ddot{V}^{A}-\vartheta \ddot{W}_{, \alpha \alpha}-l \vartheta_{\alpha}^{A} \ddot{V}_{, \alpha}^{A} \\
& \quad+K W+l^{2} K^{A} V^{A}+C \dot{W}+l^{2} C^{A} \dot{V}^{A}=P \\
& M^{A} \quad+Q_{\alpha}^{A} W_{, \alpha}+R^{A B} V^{B}+l^{2}\left(m^{A}+\widehat{m}^{A}\right) \ddot{W}+l \vartheta_{\alpha}^{A} \ddot{W}_{, \alpha}+l^{2}\left(l^{2} m^{A B}+l^{2} \widehat{m}^{A B}+\vartheta_{\alpha \beta}^{A B}\right) \ddot{V}^{B} \\
& \quad+l^{2} K^{A} W+l^{4} K^{A B} V^{B}+l^{2} C^{A} \dot{W}+l^{4} C^{A B} \dot{V}^{B}=l^{2} P^{A} \\
& -N_{\alpha \beta, \beta}+m \ddot{U}_{\alpha}+l \bar{m}^{a} \ddot{T}_{\alpha}^{a}=0 \\
& N_{\alpha}^{a}+l \bar{m}^{a} \ddot{U}_{\alpha}+l^{2} \bar{m}^{a b} \ddot{T}_{\alpha}^{b}=0
\end{aligned}
$$


Similarly to equations (4.2) and (4.3), equations (4.5) and (4.6) together with micro-macro decompositions (3.8) constitute the nonlinear tolerance model of thin visco-elastic periodic plates resting on a foundation with damping, but only for plates with the symmetry plane $z=0$. It can be observed that all above equations (4.2), (4.3) and (4.5), (4.6) have constant coefficients.

\subsection{Asymptotic model equations}

The asymptotic model equations can be obtained, from the formal point of view, using the asymptotic modelling procedure, see Woźniak et al. (2010). Below, this is done by simply neglecting terms of the order of $O\left(l^{n}\right), n=1,2, \ldots$, in equations (4.2), (4.3) and (4.5), (4.6).

Hence, from equations (4.2) and (4.3), the equations of the nonlinear asymptotic model take the form:

- constitutive equations

$$
\begin{aligned}
M_{\alpha \beta} & =-H_{\alpha \beta \gamma \delta}\left(U_{\gamma, \delta}+\frac{1}{2} W_{, \gamma} W_{, \delta}\right)-H_{\alpha \beta \gamma}^{a} T_{\gamma}^{a}+D_{\alpha \beta \gamma \delta} W_{, \gamma \delta}+D_{\alpha \beta}^{A} V^{A} \\
& -\widetilde{H}_{\alpha \beta \gamma \delta}\left[\dot{U}_{\gamma, \delta}-\frac{1}{2}\left(\dot{W}_{, \gamma} W_{, \delta}+W_{, \gamma} \dot{W}_{, \delta}\right)\right]-\widetilde{H}_{\alpha \beta \gamma}^{a} \dot{T}_{\gamma}^{a}+\widetilde{D}_{\alpha \beta \gamma \delta} \dot{W}_{, \gamma \delta}+\widetilde{D}_{\alpha \beta}^{A} \dot{V}^{A} \\
M^{A} & =-H_{\alpha \beta}^{A}\left(U_{\gamma, \delta}+\frac{1}{2} W_{, \gamma} W_{, \delta}\right)-H_{\alpha}^{a A} T_{\alpha}^{a}+D_{\alpha \beta}^{A} W_{, \gamma \delta}+D^{A B} V^{B} \\
& -\widetilde{H}_{\alpha \beta}^{A}\left[\dot{U}_{\gamma, \delta}+\frac{1}{2}\left(\dot{W}_{, \gamma} W_{, \delta}+W_{, \gamma} \dot{W}_{, \delta}\right)\right]-\widetilde{H}_{\alpha}^{a A} \dot{T}_{\alpha}^{a}+\widetilde{D}_{\alpha \beta}^{A} \dot{W}_{, \gamma \delta}+\widetilde{D}^{A B} \dot{V}^{B} \\
N_{\alpha \beta} & =B_{\alpha \beta \gamma \delta}\left(U_{\gamma, \delta}+\frac{1}{2} W_{, \gamma} W_{, \delta}\right)+B_{\alpha \beta \gamma}^{a} T_{\gamma}^{a}-H_{\alpha \beta \gamma \delta} W_{, \gamma \delta}-H_{\alpha \beta}^{A} V^{A} \\
& +\widetilde{B}_{\alpha \beta \gamma \delta}\left[\dot{U}_{\gamma, \delta}+\frac{1}{2}\left(\dot{W}_{, \gamma} W_{, \delta}+W_{, \gamma} \dot{W}_{, \delta}\right)\right]+\widetilde{a} B_{\alpha \beta \gamma}^{a} \dot{T}_{\gamma}^{a}-\widetilde{H}_{\alpha \beta \gamma \delta} \dot{W}_{, \gamma \delta}-\widetilde{H}_{\alpha \beta}^{A} \dot{V}^{A} \\
N_{\alpha}^{a} & =B_{\alpha \gamma \delta}^{a}\left(U_{\gamma, \delta}+\frac{1}{2} W_{, \gamma} W_{, \delta}\right)+B_{\alpha \gamma}^{a b} T_{\gamma}^{b}-H_{\alpha \gamma \delta}^{a} W_{, \gamma \delta}-H_{\alpha}^{a B} V^{B} \\
& +\widetilde{B}_{\alpha \gamma \delta}^{a}\left[\dot{U}_{\gamma, \delta}+\frac{1}{2}\left(\dot{W}_{, \gamma} W_{, \delta}+W_{, \gamma} \dot{W}_{, \delta}\right)\right]+\widetilde{B}_{\alpha \gamma}^{a b} \dot{T}_{\gamma}^{b}-\widetilde{H}_{\alpha \gamma \delta}^{a} \dot{W}_{, \gamma \delta}-\widetilde{H}_{\alpha}^{a B} \dot{V}^{B} \\
Q_{\alpha}^{A} & =0
\end{aligned}
$$

- equilibrium equations

$$
\begin{gathered}
M_{\alpha \beta, \alpha \beta}-\left(N_{\alpha \beta} W_{, \alpha}+Q_{\beta}^{A} V^{A}\right)_{, \beta}+(m+\widehat{m}) \ddot{W}-\vartheta \ddot{W}_{, \alpha \alpha}+K W+C \dot{W}+\theta \ddot{U}_{\alpha, \alpha}=P \\
M^{A}=0 \quad-N_{\alpha \beta, \beta}+m \ddot{U}_{\alpha}-\theta \ddot{W}_{, \alpha}=0 \quad N_{\alpha}^{a}=0
\end{gathered}
$$

where all coefficients are constant.

It can be observed that equations (4.7) and (4.8) with micro-macro decompositions (3.8) constitute the nonlinear asymptotic model of thin visco-elastic periodic plates resting on a foundation with damping for plates without the symmetry plane $z=0$.

On the other side, from equations (4.5) and (4.6), similar equations of the nonlinear asymptotic model can be derived in the form:

- constitutive equations

$$
\begin{aligned}
M_{\alpha \beta} & =D_{\alpha \beta \gamma \delta} W_{, \gamma \delta}+D_{\alpha \beta}^{A} V^{A}+\widetilde{D}_{\alpha \beta \gamma \delta} \dot{W}_{, \gamma \delta}+\widetilde{D}_{\alpha \beta}^{A} \dot{V}^{A} \\
M^{A} & =D_{\alpha \beta}^{A} W_{, \gamma \delta}+D^{A B} V^{B}+\widetilde{D}_{\alpha \beta}^{A} \dot{W}_{, \gamma \delta}+\widetilde{D}^{A B} \dot{V}^{B} \\
N_{\alpha \beta} & =B_{\alpha \beta \gamma \delta}\left(U_{\gamma, \delta}+\frac{1}{2} W_{, \gamma} W_{, \delta}\right)+B_{\alpha \beta \gamma}^{a} T_{\gamma}^{a} \\
& +\widetilde{B}_{\alpha \beta \gamma \delta}\left[\dot{U}_{\gamma, \delta}+\frac{1}{2}\left(\dot{W}_{, \gamma} W_{, \delta}+W_{, \gamma} \dot{W}_{, \delta}\right)\right]+\widetilde{B}_{\alpha \beta \gamma}^{a} \dot{T}_{\gamma}^{a} \\
N_{\alpha}^{a} & =B_{\alpha \gamma \delta}^{a}\left(U_{\gamma, \delta}+\frac{1}{2} W_{, \gamma} W_{, \delta}\right)+B_{\alpha \gamma}^{a b} T_{\gamma}^{b}+\widetilde{B}_{\alpha \gamma \delta}^{a}\left[\dot{U}_{\gamma, \delta}+\frac{1}{2}\left(\dot{W}_{, \gamma} W_{, \delta}+W_{, \gamma} \dot{W}_{, \delta}\right)\right]+\widetilde{B}_{\alpha \gamma}^{a b} \dot{T}_{\gamma}^{b} \\
Q_{\alpha}^{A} & =0 \quad R^{A B}=0
\end{aligned}
$$


- equilibrium equations

$$
\begin{aligned}
& M_{\alpha \beta, \alpha \beta}-\left(N_{\alpha \beta} W_{, \alpha}\right)_{, \beta}+(m+\widehat{m}) \ddot{W}-\vartheta \ddot{W}_{, \alpha \alpha}+K W+C \dot{W}=P \\
& M^{A}=0 \quad-N_{\alpha \beta, \beta}+m \ddot{U}_{\alpha}=0 \quad N_{\alpha}^{a}=0
\end{aligned}
$$

with all coefficients constant.

It is necessary to observe that equations (4.9) and (4.10) and micro-macro decompositions (3.8) constitute the nonlinear asymptotic model of thin visco-elastic periodic plates resting on a foundation with damping for plates with the symmetry plane $z=0$.

\section{Final remarks}

A new nonlinear non-asymptotic model for dynamic problems of thin visco-elastic periodic plates resting on a foundation with damping is proposed in this note. This model is based on the assumptions of von Kármán nonlinear thin plate theory. In order to derive the model governing equations, the tolerance modelling is applied.

Summarizing, it can be concluded that:

- The proposed approach replaces governing equations of plates having highly oscillating, periodic, non-continuous functional coefficients by the model equations with constant coefficients, which can be solved using suitable well-known methods. Thus, the nonlinear tolerance model can be a useful tool in investigations of various dynamic phenomena of the considered plate structures.

- In contrast to the original formulations, the new proposed nonlinear tolerance model introduces some averaged, effective properties of the plate structure.

- Dynamic behaviour of the plates under consideration is described in this model by some new unknowns as averaged deflections (macrodeflections) and averaged in-plane displacements (in-plane macrodisplacements) and amplitudes of their disturbances due to inhomogeneity of the structure. These new kinematic unknowns have to be slowly-varying functions in $\mathbf{x}$, which constitutes conditions of physical reliability of the solutions.

- The very important feature of the proposed nonlinear tolerance model is that its governing equations involve terms with the microstructure parameter. Hence, this tolerance model makes it possible to investigate the effect of the microstructure size on the overall dynamic behaviour of thin visco-elastic periodic plates resting on a foundation with damping in the framework of von Kármán nonlinear thin plate theory. Using this model, some phenomena in dynamic problems caused by the internal periodic structure of the plates under consideration can be investigated.

- It can be observed that the transition from the governing equations of geometrically nonlinear tolerance models to the equations of the linear tolerance models may take place on two levels - micro, when the effect of nonlinear terms with fluctuation amplitudes is omitted, and micro-macro, when all nonlinear terms are neglected.

- It should be noted that the proposed model is a kind of generalization in relation to the known tolerance models shown by Domagalski and Jędrysiak (2012, 2015), where some bending nonlinear problems of thin periodic plates were described. Domagalski and Jędrysiak (2014) analysed nonlinear vibrations of slender periodic beams resting on a foundation with damping, wherreas Marczak and Jędrysiak (2014) investigated damped vibrations of plate strips with periodically distributed concentrated masses.

Various applications of the proposed tolerance and asymptotic models to dynamics of thin visco-elastic periodic plates with moderately large deflections resting on a foundation with damping will be analysed in the forthcoming papers. 


\section{References}

1. Awrejcewicz J., Kurpa L., Shmatko T., 2013, Large amplitude free vibration of orthotropic shallow shells of complex shapes with variable thickness, Latin American Journal of Solids and Structures, 10, 149-162

2. Brito-Santana H., Wang Y.S., Rodrguez-Ramos R., Bravo-Castillero J., GuinovartDíaz R., Volnei Tita, 2015, A dispersive nonlocal model for shear wave propagation in laminated composites with periodic structures, European Journal of Mechanics - A/Solids, 49, 35-48

3. Camier C., Touzé C., Thomas O., 2009, Non-linear vibrations of imperfect free-edge circular plates and shells, European Journal of Mechanics - A/Solids, 28, 500-515

4. Dallot J., Sab K., Foret G., 2009, Limit analysis of periodic beams, European Journal of Mechanics - A/Solids, 28, 166-178

5. De Carvalho N.V., Pinho S.T., Robinson P., 2011, Reducing the domain in the mechanical analysis of periodic structures, with application to woven composites, Composites Science and Technology, 71, 969-979

6. Domagalski Ł., JęDrysiak J., 2012, On the elastostatics of thin periodic plates with large deflections, Meccanica, 41, 1659-1671

7. Domagalski Ł., JęDrysiak J., 2014, Nonlinear vibrations of periodic beams, Journal of Vibrations in Physical Systems, 26, 73-78

8. Domagalski Ł., JęDrysiak J., 2015, On the tolerance modelling of geometrically nonlinear thin periodic plates, Thin-Walled Structures, 87, 183-190

9. Fallah A., Aghdam M.M., 2011, Nonlinear free vibration and post-buckling analysis of functionally graded beams on nonlinear elastic foundation, European Journal of Mechanics - A/Solids, 30, 571-583

10. Golmakani M.E., Alamatian J., 2013, Large deflection analysis of shear deformable radially functionally graded sector plates on two-parameter elastic foundations, European Journal of Mechanics - A/Solids, 42, 251-265

11. Gupta A.K., Khanna A., Gupta D.V., 2009, Free vibration of clamped visco-elastic rectangular plate having bi-direction exponentially thickness variations, Journal of Theoretical and Applied Mechanics, 47, 2, 457-471

12. He W.M., Chen W.Q., Qiao H., 2013, Two-scale analytical solutions of multilayered composite rectangular plates with in-plane small periodic structure, European Journal of Mechanics A/Solids, 40, 123-130

13. Houmat A., 2013, Nonlinear free vibration of laminated composite rectangular plates with curvilinear fibers, Composites Structures, 106, 211-224

14. HuAnG J.Y., 2004, Uniformly valid asymptotic solutions of the nonlinear unsymmetrical bending for orthotropic rectangular thin plate of four clamped edges with variable thickness, Applied Mathematics and Mechanics, 25, 817-826

15. Jasion P., Magnucka-Blandzi E., Szyc W., Magnucki K., 2012, Global and local buckling of sandwich circular and beam-rectangular plates with metal foam core, Thin-Walled Structures, 61, 154-161

16. JEDRYSIAK J., 2000, On the stability of thin periodic plates, European Journal of Mechanics A/Solids, 19, 3, 487-502

17. JĘDRYSIAK J., 2003, Free vibrations of thin periodic plates interacting with an elastic periodic foundation, International Journal of Mechanical Sciences, 45, 8, 1411-1428

18. JęDrysiak J., 2009, Higher order vibrations of thin periodic plates, Thin-Walled Structures, 47, 890-901 
19. JĘDrYSIAK J., 2013, Modelling of dynamic behaviour of microstructured thin functionally graded plates, Thin-Walled Structures, 71, 102-107

20. JęDrysiak J., Michalak B., 2011, On the modelling of stability problems for thin plates with functionally graded structure, Thin-Walled Structures, 49, 627-635

21. JęDrysiak J., PAŚ A., 2014, Dynamics of medium thickness plates interacting with a periodic Winkler's foundation: non-asymptotic tolerance modelling, Meccanica, 49, 1577-1585

22. KAŹMierczaK M., JęDrysiak J., 2011, Tolerance modelling of vibrations of thin functionally graded plates, Thin-Walled Structures, 49, 1295-1303

23. Kohn R.V., Vogelius M., 1984, A new model of thin plates with rapidly varying thickness, International Journal of Solids and Structures, 20, 333-350

24. Królak M., Kowal-Michalska K., Mania R.J., Świniarski J., 2009, Stability and load carrying capacity of multi-cell thin-walled columns of rectangular cross-sections, Journal of Theoretical and Applied Mechanics, 47, 1, 435-456

25. Lei Y., Murmu T., Adhikari S., Friswell M.I., 2013, Dynamic characteristics of damped viscoelastic nonlocal Euler-Bernoulli beams, European Journal of Mechanics - A/Solids, 42, 125136

26. LEVy S., 1942, Bending of rectangular plates with large deflections, NACA Rep No. 737. NACA Tech Note No. 846

27. Lurie S.A., Belov P.A., Tuchkova N.P., 2005, The application of the multiscale models for description of the dispersed composites, Composites Part A: Applied Science and Manufacturing, 36, 2, 145-152

28. MagnuckA-Blandzi E., 2010, Non-linear analysis of dynamic stability of metal foam circular plate, Journal of Theoretical and Applied Mechanics, 48, 1, 207-217

29. Mahmoudkhani S., Haddadpour H., Navazi H.M., 2014, The effects of nonlinearities on the vibration of viscoelastic sandwich plates, nternational Journal of Non-Linear Mechanics, 62, 41-57

30. Manevich A., KoŁakowski Z., 2011, Free and forced oscillations of Timoshenko beam made of viscoelastic material, Journal of Theoretical and Applied Mechanics, 49, 1, 3-16

31. Marczak J., JęDrysiak J., 2014, Analysis of vibrations of plate strip with concentrated masses using tolerance averaging technique, Journal of Vibrations in Physical Systems, 26, 161-168

32. Matysiak S.J., Perkowski D.M., 2014, Temperature distributions in a periodically stratified layer with slant lamination, Heat Mass Transfer, 50, 75-83

33. Meenen J., Altenbach H., 2001, A consistent deduction of von Kármán-type plate theories from three-dimensional nonlinear continuum mechanics, Acta Mechanica, 147, 1-17

34. MichalaK B., 2001, The meso-shape functions for the meso-structural models of wavy-plates, ZAMM, 81, 639-641

35. NAGórko W., Woźniak C., 2002, Nonasymptotic modelling of thin plates reinforced by a system of stiffeners, Electronic Journal of Polish Agricultural Universities - Civil Engineering, 5, 2

36. Perliński W., Gajdzicki M., Michalak B., 2014, Modelling of annular plates stability with functionally graded structure interacting with elastic heterogeneous subsoil, Journal of Theoretical and Applied Mechanics, 52, 2, 485-498

37. Reinhall P.G., Miles R.N., 1989, Effect of damping and stiffness on the random vibration of non-linear periodic plates, Journal of Sound and Vibration, 132, 33-42

38. Saha G.C., Kalamkarov A.L., Georgiades A.V., 2007, Effective elastic characteristics of honeycomb sandwich composite shells made of generally orthotropic materials, Composites Part A: Applied Science and Manufacturing, 38, 6, 1533-1546

39. Schmitz A., Horst P., 2014, A finite element unit-cell method for homogenised mechanical properties of heterogeneous plates, Composites Part A: Applied Science and Manufacturing, 61, $23-32$ 
40. Singha M.K., Rupesh Daripa, 2009, Nonlinear vibration and dynamic stability analysis of composite plates, Journal of Sound and Vibration, 328, 541-554

41. Teter A., 2011, Dynamic critical load based on different stability criteria for coupled buckling of columns with stiffened open cross-sections, Thin-Walled Structures, 49, 589-595

42. Timoshenko S., Woinowsky-Krieger S., 1959, Theory of Plates and Shells, McGraw-Hill, New York

43. TomczyK B., 2007, A non-asymptotic model for the stability analysis of thin biperiodic cylindrical shells, Thin-Walled Structures, 45, 941-944

44. Vlasov W.Z., Leontiev N.N., 1960, Beams, Plates and Shells on Rigid Subsoil (in Russian), Gos. Izd. Fiz.-Mat. Lit., Moskva

45. WierzBicki E., Woźniak C., 2000, On the dynamics of combined plane periodic structures, Archive of Applied Mechanics, 70, 6, 387-398

46. Wierzbicki E., Woźniak C., Woźniak M., 2001, On the modelling of transient micro-motions and near-boundary phenomena in a stratified elastic layer, International Journal of Engineering Science, 39, 13, 1429-1441

47. Woźniak C. (EDit.), 2001, Mechanics of Elastic Plates and Shells (in Polish), PWN, Warsaw

48. Woźniak C., et al. (EDIT.), 2010, Mathematical Modelling and Analysis in Continuum Mechanics of Microstructured Media, Silesian Techn. Univ. Press, Gliwice

49. Woźniak C., Michalak B., Jędrysiak J. (EDit.), 2008, Thermomechanics of Microheterogeneous Solids and Structures. Tolerance Averaging Approach, Lodz Univ. Techn. Press, Lodz

50. Yajuvindra Kumar, Lal R., 2013, Prediction of frequencies of free axisymmetric vibration of two-directional functionally graded annular plates on Winkler foundation, European Journal of Mechanics - A/Solids, 42, 219-228

51. Yaghoobi H., Torabi M., 2013, An analytical approach to large amplitude vibration and postbuckling of functionally graded beams rest on non-linear elastic foundation, Journal of Theoretical and Applied Mechanics, 51, 1, 39-52

52. Youzera H., Meftah S.A., Challamel N., Tounsi A., 2012, Nonlinear damping and forced vibration analysis of laminated composite beams, Composites Part B: Engineering, 43, 1147-1154

53. Zhou X.Q., Yu D.Y., Shao X., Wang S., Tian Y.H., 2014, Band gap characteristics of periodically stiffened-thin-plate based on center-finite-difference-method, Thin-Walled Structures, 82, 115-123 